\title{
Erfassung und Quantifizierung von Geruchsimmissionen durch Kombination hochempfindlicher Sensorsysteme mit einem Geruchsnetzwerk
}

\author{
Wolfhard Reimringer ${ }^{1}$, Thorsten Conrad ${ }^{1}$, Andreas Schütze $^{2}$ \\ ${ }^{1} 3 \mathrm{~S} \mathrm{GmbH}$, Mainzer Str. 148, 66121 Saarbrücken \\ 2Universität des Saarlandes, Lehrstuhl für Messtechnik, Campus A5.1, 66123 Saarbrücken \\ reimringer@3S-ing.de, conrad@3S-ing.de, schuetze@LMT.uni-saarland.de
}

\section{Zusammenfassung}

Die Belästigung von Anwohnern durch Emissionen von Industriebetrieben wird abhängig von der Art der Emission nach unterschiedlichen Verfahren bewertet. Während für regulierte Schadstoffe Referenzmesstechnik existiert, die die Einhaltung festgelegter Grenzwerte im Sinne von Mittelwerten überwacht, werden Gerüche humansensorisch unter Beachtung einschlägiger Normen beurteilt. Im Falle großflächig verteilter Quellen und weit entfernter Immissionsorte ist der Aufwand einer Rasterbegehung immens und wird durch sporadisches Auftreten der Belästigung weiter erschwert.

Eine technische Lösung zur Geruchsüberwachung kann die menschliche Nase zwar nicht ersetzen, als Unterstützung für das anerkannte humansensorische Verfahren wäre es jedoch wünschenswert, eine kontinuierliche Indikativmessung zu realisieren. Diese muss eine für den Immissionsbereich ausreichende Empfindlichkeit aufweisen und gleichzeitig preiswert genug sein um damit ein engmaschiges Messnetz aufbauen zu können.

Das im Folgenden beschriebene Pilotprojekt umfasst sowohl Gassensorsysteme im Immissionsbereich als auch die menschliche Referenz: Messstationen auf Basis temperaturzyklisch betriebener Metalloxidsensoren wurden in einem Gebiet mit Radius von $10 \mathrm{~km}$ um die vermutete Quelle positioniert. Gleichzeitig wurde durch ein Geruchsnetzwerk aus Anwohnern eine Metadatenbasis aufgenommen, um Referenzevents für das Training der Auswertealgorithmen für die Sensordaten zu gewinnen.

Insgesamt konnte gezeigt werden, dass mit dem gewählten Vorgehen eine Korrelation von Sensordaten mit dem betrachteten Zielgeruch auch im Immissionsbereich grundsätzlich möglich ist. Zukünftige Arbeiten werden den Ausbau der notwendigen Infrastruktur für die Datenanbindung und -auswertung sowie die Steigerung der Messempfindlichkeit umfassen.

Keywords: Gassensorik, Immission, Sensornetzwerk, Geruch, Bürgerbeteiligung

\section{Hintergrund}

Die zuverlässige und objektive Erfassung von Gerüchen stellt in vielen Bereichen nach wie vor eine große Herausforderung für moderne Messsysteme dar. Hintergrund ist die Komplexität von Gerüchen einerseits, die sich vielfach aus einem Cocktail vieler verschiedener Substanzen zusammensetzen, und der subjektive Eindruck andererseits, der vom Menschen, seiner Erfahrung und seinem Gesundheitszustand abhängt [1]. Eine objektive Bewertung von Gerüchen erfolgt mittels Olfaktometrie [2], wofür ein Panel von normgerecht riechenden Probanden erforderlich ist, so dass weder online noch kontinuierlich gemessen werden kann. Während für Geruchsbewertungen im Labor durchaus entsprechende Messsysteme demonstriert wurden [3], teilweise auch für die Bewertung von Geruchsemmissionen an der
Quelle, z. B. bei Mülldeponien [4], stellt die Erfassung und Quantifizierung von Geruchsimmissionen angesichts deutlich geringerer Konzentrationen und sehr komplexer Hintergrundmatrix eine große Herausforderung für die Forschung dar. Motivation für das hier präsentierte Projekt waren anhaltende Geruchsbeschwerden von Anwohnern des Warndt, eines waldreichen Gebiets im Saarland an der Grenze zu Frankreich (siehe auch Abb. 1). Als Geruchsquelle wurde dort die sogenannte Chemie-Plattform Carling in unmittelbarer Grenznähe auf französischer Seite vermutet. Messungen mit konventionellen Methoden gemäß den Vorgaben der TA Luft bzw. des BImSchG ergaben keine auffälligen Befunde, allerdings werden damit auch keine Geruchsstoffe erfasst. Im Jahr 2014 beauftragte das saarländische Umweltministerium die $3 \mathrm{~S} \mathrm{GmbH}$ daraufhin mit einer Studie, bei der die Geruchsbelastungen in einer Messkampagne 
mit hochempfindlichen Sensorsystemen erfasst werden sollten. Die Messungen wurden mit einem Geruchsnetzwerk verknüpft, um die Wahrnehmungen der Anwohner als Referenz nutzen zu können.

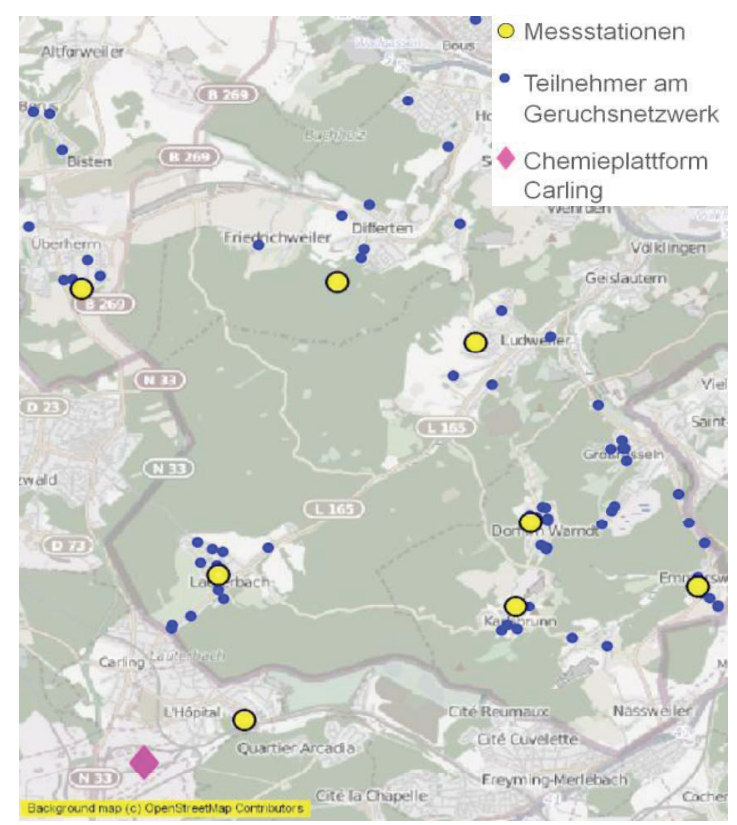

Abb. 1: Warndtgebiet mit der vermuteten Geruchsquelle, den Messstationen sowie den Teilnehmern des Geruchsnetzwerks. Kartenausschnitt ca. $10 \times 15 \mathrm{~km}^{2}$.

\section{Experimentelles}

Für die Messungen wurden Sensorsysteme modifiziert, die üblicherweise in der industriellen Qualitätskontrolle z. B. zur Erfassung von Fehlgerüchen bei Kunststoffen oder Kosmetika eingesetzt werden. Mit einer Pumpe wird dabei Zuluft angesaugt und nach Staubfilterung über zwei Halbleitergassensoren geführt.

Die Sensoren werden zur Verbesserung der Selektivität, aber auch der Empfindlichkeit und Stabilität temperaturzyklisch betrieben, d. h. die Sensortemperatur wird periodisch variiert, so dass sich je nach Gasatmosphäre charakteristische Signalmuster ergeben, die über komplexe Mustererkennungsalgorithmen klassifiziert werden [5-7].

Zusätzlich ist im System ein Feuchte- und Temperatursensor integriert, um die Umgebungsbedingungen zu erfassen. Weiterhin ist der Anschluss eines Windsensors möglich, um auch Windrichtung und -geschwindigkeit protokollieren zu können. Alle Daten werden auf einer SD-Karte gespeichert, die eine kontinuierliche Erfassung über mehrere Wochen erlaubt, das Rohdatenaufkommen beträgt etwa $2 \mathrm{~GB}$ pro Monat. In der Projektlaufzeit von über einem Jahr waren acht
DOI 10.5162/sensoren2016/5.2.4 Sensorsysteme installiert, wobei keinerlei Ausfälle beobachtet wurden.
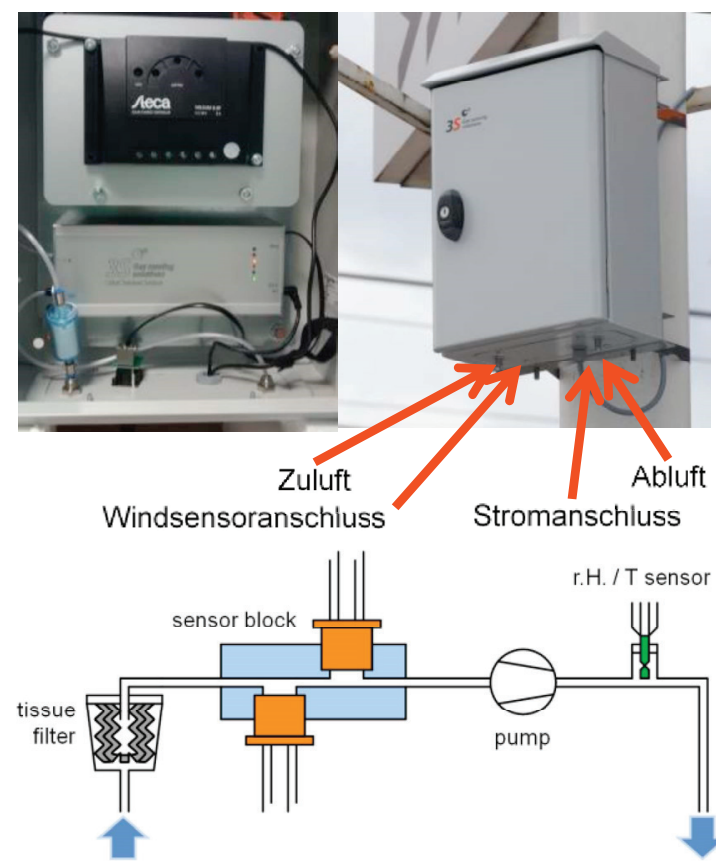

Abb. 2: Messsystem zur Geruchserfassung in der Außenluft, basierend auf zwei Halbleitergassensoren. Oben rechts: Außenansicht des Messsystems, oben links: Innenansicht mit Spannungsversorgung (oben) und Messhardware (unten). Unten: Messpfad mit Partikelfilter, Sensoren, Pumpe und Temperatur-FeuchteSensor.

\section{Geruchsnetzwerk}

$\mathrm{Zu}$ Beginn des Projektes wurde an alle Bewohner des Warndtgebietes ein Fragebogen in Anlehnung an die VDI-Richtlinie 3883 [8] verteilt, um die Ist-Situation zu erfassen. Hieraus konnten Informationen zu typischen Zeiten einer Geruchsbelästigung (morgens und abends), Wettereinfluss (Südwest-Wind, Inversionswetterlage), Geruchsqualität (chemisch-süßlich, stechend) sowie der Positionierung der Sensorsysteme gewonnen werden.

Zusätzlich wurde in den Fragebögen die Bereitschaft zur freiwilligen Teilnahme an begleitenden Beobachtungen in einem Geruchsnetzwerk abgefragt. Etwa 50 Anwohner aus 8 Orten beteiligten sich letztlich am Geruchsnetzwerk zur objektiven Erfassung der vorliegenden Geruchsbelastungen. Über eine Webseite sollten mindestens zweimal täglich die beobachteten Gerüche nach Art und Stärke gemeldet werden, wobei das Hauptaugenmerk auf stechend chemischen bzw. süßlichen Gerüchen (Zielgeruch) lag. 
Tab. 1: Geruchsmeldungen in \% bezogen auf die Gesamtmeldungen der jeweiligen Phase und Windrichtung. SW-Wind: Sektor 170-280', andere Windrichtung: $280-170^{\circ}$ Intensitäten von 1 (kaum wahrnehmbar) bis 6 (unerträglich)

\begin{tabular}{|c|c|c|c|c|}
\hline & \multicolumn{2}{|c|}{ Phase I } & \multicolumn{2}{|c|}{ Phase II } \\
\hline & SW & andere & SW & andere \\
\hline $\begin{array}{l}\text { Ziel- } \\
\text { geruch } \\
\text { gesamt }\end{array}$ & 9,7 & 9,0 & 13,0 & 9,9 \\
\hline $5-6$ & 1,6 & 1,6 & 5,4 & 3,7 \\
\hline $3-5$ & 4,8 & 3,9 & 5,2 & 4,4 \\
\hline $1-2$ & 3,4 & 3,5 & 2,4 & 1,8 \\
\hline $\begin{array}{c}\text { Null- } \\
\text { geruch }\end{array}$ & 78,2 & 76,3 & 82,9 & 84,7 \\
\hline $\begin{array}{l}\text { Fremd- } \\
\text { geruch }\end{array}$ & 12,2 & 14,7 & 4,1 & 5,4 \\
\hline
\end{tabular}

Insgesamt gingen 8500 Meldungen ein, mit Beobachtungszeiträumen im Winter 2014/2015 (Phase I) und im Sommer 2015 (Phase II). Wesentlich für die Entscheidung, eine zweite Erhebungsphase durchzuführen, war die geringe Dichte an Meldungen in Phase I; in Phase II konnte eine Steigerung von über $100 \%$ verzeichnet. Zusätzlich konnte so der von den Betroffenen reklamierte jahreszeitliche Unterschied untersucht werden.

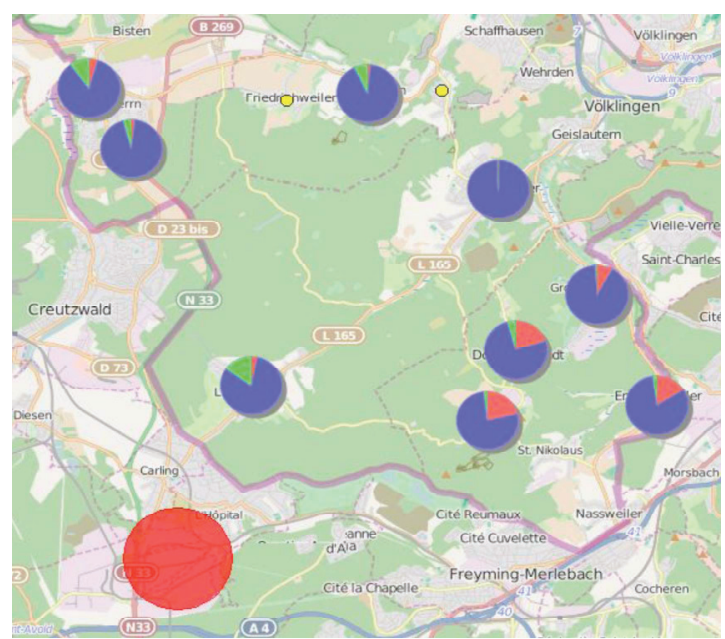

Abb. 3: Relative Verteilung von Nullgeruchsmeldungen (blau), Fremdgeruch (grün) und Zielgeruch (rot) in den verschiedenen Orten. Roter Kreis: Chemieplattform Carling als vermutete Quelle.

Aus Tab. 1 ist ersichtlich, dass im Winter der Anteil an Fremdgeruchsmeldungen deutlich höher ist. Dies korreliert mit Kommentaren und Beschwerden hinsichtlich Belästigung durch private Feuerungseinrichtungen. Im Sommer hingegen ist der Zusammenhang zwischen
DOI 10.5162/sensoren2016/5.2.4 Zielgeruch und Windrichtung sowie der Anteil an stark belästigenden Zielgeruchssituationen stärker ausgeprägt.

Eine ortsbezogene Analyse ergab, dass in einem eng begrenzten geographischen Bereich eine Häufung des Zielgeruchs registriert wurde (Abb. 3). Dieser Bereich liegt im Nordosten der vermuteten Quelle, was in Verbindung mit einer Hauptwindrichtung aus Südwest plausibel erscheint (vgl. Abb. 4).

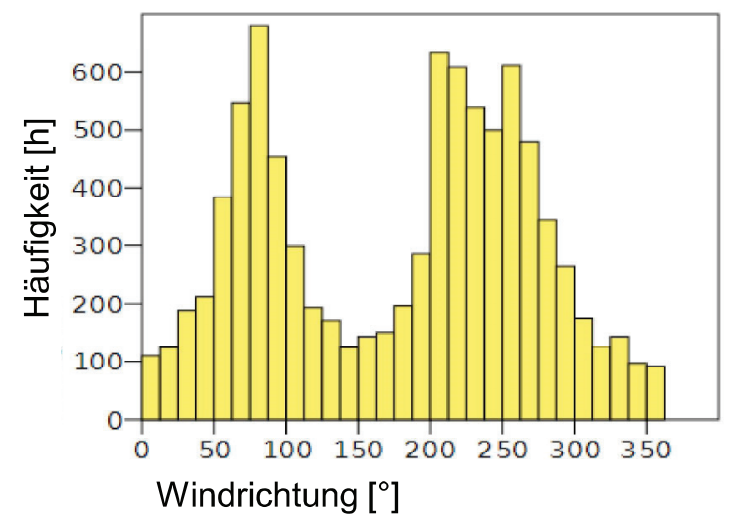

Abb. 4: Verteilung der Windrichtung (Stundenmitte/werte) für das Jahr 2014 am Beobachtungsort L'Hôpital

\section{Geruchsevents als Trainingsreferenz}

Die Gewinnung von Referenzdaten für das Sensortraining aus den Geruchsmeldungen stellte eine besondere Herausforderung dar: Die zeitliche Streuung der Rückmeldungen wurde durch die Vorgabe von Zeitfenstern am Morgen und am Abend abzufangen versucht, jedoch konnten nicht alle der freiwilligen Melder diese Zeiten einhalten. Zusätzlich ergaben sowohl die unterschiedlichen Beobachtungspositionen an den jeweiligen Wohnadressen als auch Unterschiede in der persönlichen Geruchswahrnehmung ein uneinheitliches Bild der Situation.

Zur Bewertung der Geruchssituation wurden die Einzelmeldungen zu sogenannten Geruchsevents zusammengefasst. Ein Event ist eine zeitliche Zusammenfassung mehrerer Meldungen und gibt an, ob im betrachteten Zeitfenster mehr Zielgeruch, Fremdgeruch oder Nullgeruch beobachtet wurde. Dieser integrative Ansatz verringert einerseits die Unsicherheit der Bewertung, durch die Dauer des betrachteten Zeitfensters umfasst dieses unter Umständen aber auch unterschiedliche Situationen am Sensor.

In Phase I wurden Geruchsevents manuell gesucht, indem die Meldungen in einem ZeitEntfernungsdiagramm (Abb. 5) dargestellt und gesichtet wurden. Die Meldungen sind nach der betrachteten Geruchsart (Zielgeruch) 
gefiltert und anhand der gemeldeten Intensität farblich codiert.

Grundidee ist hier, dass bei einem Emissionsereignis die Belästigung mit zeitlicher Staffelung in immer größerer Entfernung zur Quelle wahrgenommen wird bis die Intensität der Immission schließlich unter die Wahrnehmungsschwelle sinkt. Somit müsste ein Geruchsereignis durch eine Häufung von Zielgeruchsmeldungen in zeitlicher Nähe und im Bereich kleiner Entfernungen zur Quelle auffallen. Eine wesentliche Schwierigkeit bei diesem Vorgehen ist die nicht per se berücksichtigte Richtung der Ausbreitung durch den großen Winkelabschnitt betrachteter Immissionsorte kommt es zwangsläufig zur Vermischung belasteter und unbelasteter Orte in der Darstellung nach Abb. 5, so dass letztlich die Beschränkung auf eine Hauptwindrichtung gemäß Abb. 4 notwendig wurde.

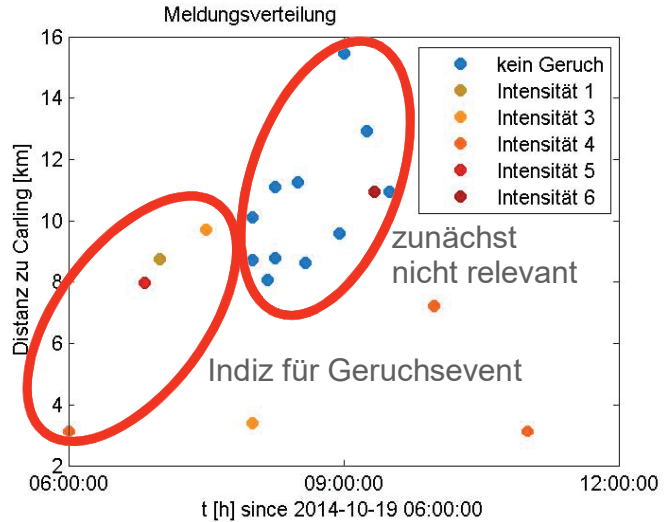

Abb. 5: Manuelle Untersuchung von Geruchsmeldungen auf ein konsistentes, sich ausbreitendes Geruchsevent mithilfe von Zeit und Entfernung von der vermuteten Quelle.

Für Phase II wurde zur Behandlung der deutlich dichteren Datenlage eine automatische Eventsuche entwickelt, die für die Eventidentifikation Zeitabschnitte von sechs Stunden verwendet. Die so ermittelten Referenzzeiten wurden anschließend manuell auf den Häufungspunkt der jeweiligen Geruchsart beschränkt.

Die Qualität der Events für das Sensortraining wurde zudem gesteigert, indem nur eindeutige Situationen zugelassen wurden. Ein wichtiger Schritt war die Beschränkung auf den am meisten betroffenen geographischen Bereich. Zusätzlich wurden Nullgeruchsevents bevorzugt, bei denen der Wind seit mindestens zwei Stunden mit messbarer Geschwindigkeit nicht aus Richtung der vermuteten Quelle wehte (andere Windrichtung), Zielgeruchsevents wurden bevorzugt mit Südwest-Wind ausgewählt.
Tab. 2: Identifizierte Geruchsevents

\begin{tabular}{|c|c|c|c|}
\hline & Phase I & \multicolumn{2}{|c|}{ Phase II } \\
gesamt & Training \\
\hline $\begin{array}{c}\text { Ziel- } \\
\text { geruch }\end{array}$ & 2 & 32 & 15 \\
\hline $\begin{array}{c}\text { Null- } \\
\text { geruch }\end{array}$ & $2^{*}$ & 100 & 15 \\
\hline $\begin{array}{c}\text { Fremd- } \\
\text { geruch }\end{array}$ & 1 & 4 & 1 \\
\hline Summe & 5 & 136 & 31 \\
\hline
\end{tabular}

${ }^{*}$ keine weiteren gesucht

Tab. 2 zeigt die Anzahl der identifizierten Geruchsevents im Überblick, wobei die gute Rückmeldelage in Phase II neben der automatischen Suche eine Aufteilung in Trainings- und Validierungsevents ermöglichte.

\section{Sensortraining und Ergebnisse}

Der Informationsgehalt temperaturzyklisch betriebener Halbleitergassensoren liegt in den Formmerkmalen der Sensorreaktion (vgl. Abb. 6). Für die Auswertung der Sensorrohdaten wurden zunächst Messungen aus den Zeiträumen der gefundenen Trainingsevents herangezogen und die Formmerkmale der temperaturzyklisch betriebenen Sensoren einem überwachten Lernverfahren (Lineare Diskriminanzanalyse, LDA) zugeführt.

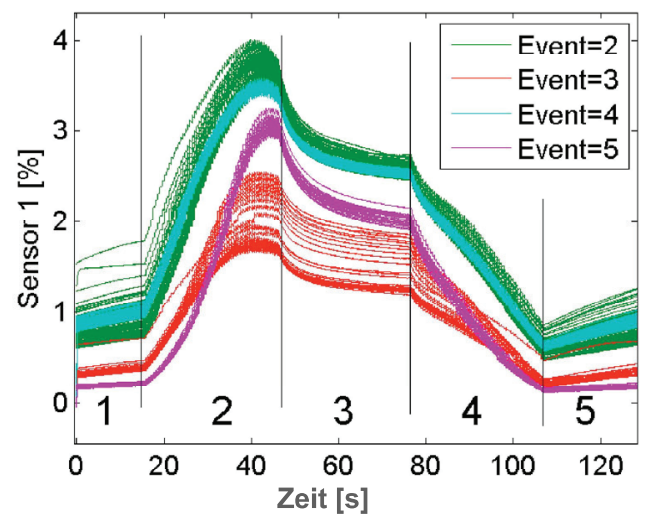

Abb. 6: Formänderung der Sensorreaktion eines temperaturzyklisch betriebenen Halbleitergassensors (UST GGS 1330) für verschiedene Events, eingeteilt in charakteristische Abschnitte 1-5.

Hierbei konnten die Eventarten für den makrostrukturierten Sensor vom Typ UST GGS 1330 nicht zufriedenstellend getrennt werden, vgl. Abb. 7. Als mögliche Gründe kommen eine für die Zielstoffe nicht ausreichend empfindliche Sensorschicht sowie die große thermische Trägheit dieses auf Keramiksubstrat 
aufgebauten Sensors infrage - aus letzterem resultiert ein mit $180 \mathrm{~s}$ Dauer vergleichsweise langer Temperaturzyklus, während dessen Ablauf die aus der Umgebung angesaugte Zusammensetzung der Zielkomponenten nicht konstant bleibt und somit die Sensorantwort nicht erwartungsgemäß beeinflusst.

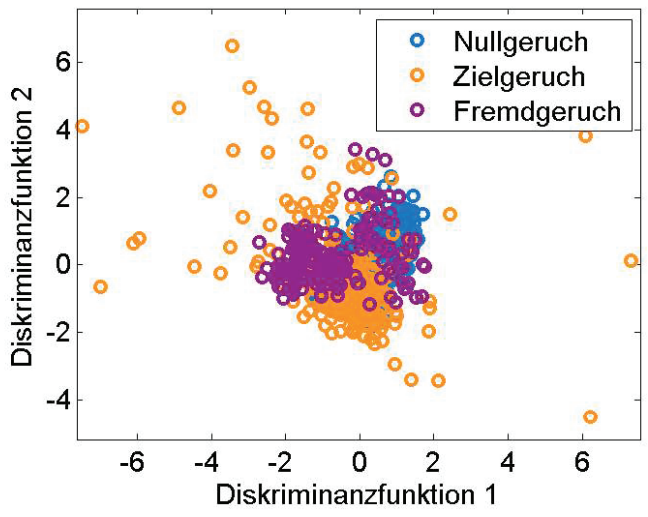

Abb. 7: $\quad$ Trennung der Messungen (UST GGS 1330) am Standort Dorf im Warndt in Phase I.

Für den mikrostrukturierten Sensor AS MLV lieferte die LDA bessere Ergebnisse. Hier konnte ein Zyklus mit nur 20 s Dauer verwendet werden. Abb. 8 zeigt die Trennung exemplarisch für den Standort Dorf im Warndt, die Trennung erfolgt hier entlang Diskriminanzfunktion 1.

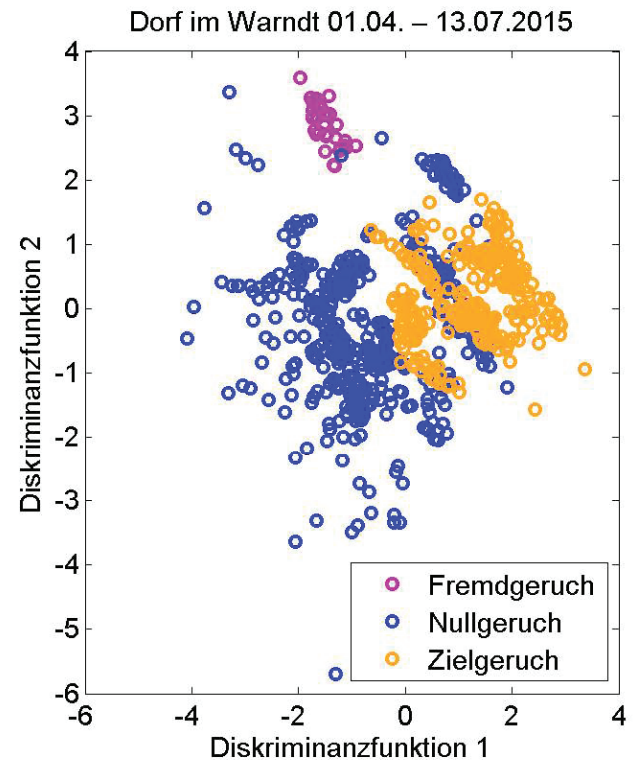

Abb. 8: Exemplarische Trennung der Messungen (AS MLV) am Standort Dorf im Warndt für die 31 Trainingsevents in Phase II. Die Trennung erfolgt hier entlang Diskriminanzfunktion 1.
DOI 10.5162/sensoren2016/5.2.4

Der zeitliche Verlauf des Funktionswertes der trennenden Diskriminanzfunktion wurde anschließend für alle Einzelmessungen berechnet und zur anschaulichen Betrachtung bzw. Validierung des Trainings herangezogen.

Abb. 9 zeigt eine die Auswertung am Standort Dorf im Warndt über eine Woche, in der vom Netzwerk drei Zielgeruchsevents (rot hinterlegt) sowie neun Nullgeruchsereignisse (grün hinterlegt) gemeldet wurden. Fünf der gezeigten Events wurden im Training verwendet, anhand der verbleibenden sieben Events (blaue Pfeile) kann das Training validiert werden.

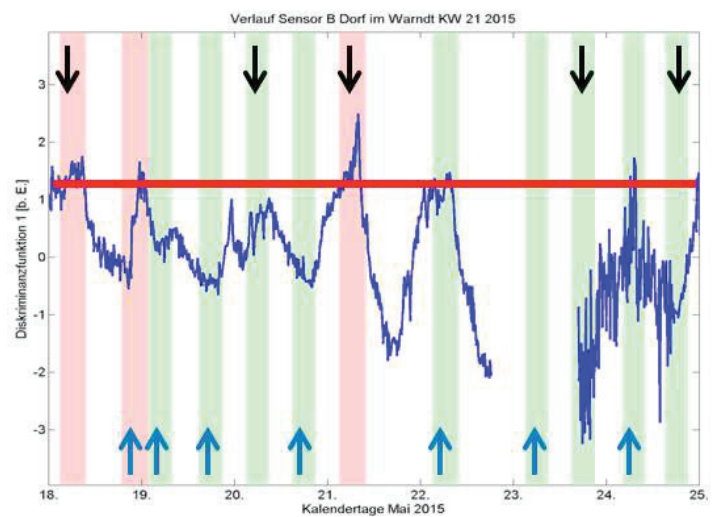

Abb. 9: Online-Darstellung der Zielgeruchsintensität für den Ort Dorf im Warndt, 21. KW 2015, mit möglicher Warnschwelle (rote Linie). Referenzevents farbig hinterlegt (rot: Zielgeruch, grün: Nullgeruch), aufgeteilt für Training (schwarze Pfeile) und Validierung (blaue Pfeile)

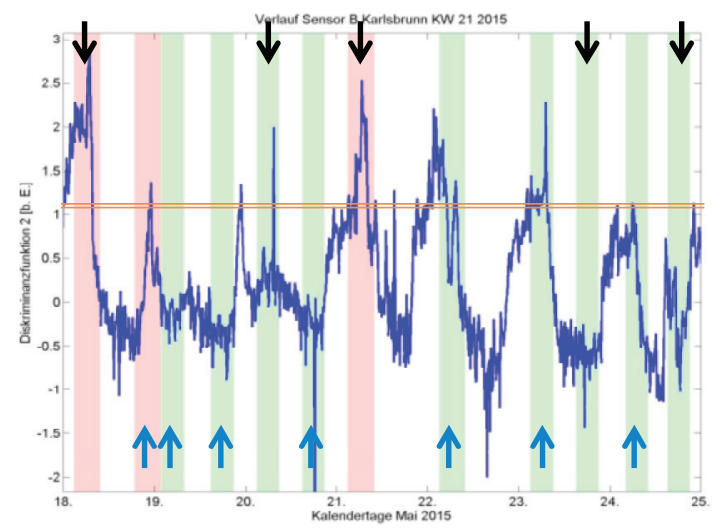

Abb. 10: Online-Darstellung der Zielgeruchsintensität für den Ort Karlsbrunn, 21. KW 2015, Notation s. Abb. 9.

Es zeigt sich, dass das trainierte System in Verbindung mit einer geeigneten Warnschwelle alle Zielgeruchsevents erkennen kann. Jedoch kommt es auch zu Fehlalarmen, also der Bewertung von Nullgeruchssituationen als 
Zielgeruch. Insgesamt weist der Verlauf einen Tagesgang auf, der auf weitere Einflussfaktoren hindeutet, die einem Tag-Nacht-Rhythmus unterworfen sind und das Grundniveau der detektierten Stoffe beeinflussen. Der temperaturzyklische Betrieb wird durch Änderungen von Temperatur und Luftfeuchte aufgrund der geregelten Heizung des Sensors und des Charakters der Datenvorverarbeitung nicht direkt gestört, so dass eine relevante Wechselwirkung zwischen Witterung und der atmosphärischen Verteilung gasförmiger Stoffe zu vermuten ist. Der Versuch einer rechnerischen Kompensation des Sensorsignals anhand der Messwerte für Temperatur- und Feuchte lieferte erwartungsgemäß keine brauchbaren Resultate. Der Einfluss von Globalstrahlung und Niederschlag konnte mangels verfügbarer Messwerte für das betrachtete Gebiet nicht näher untersucht werden.

Als weitere Erkenntnis lässt sich beobachten, dass lokale Einflüsse sich auf einer kürzeren Zeitskala abbilden als regionale Ereignisse. Während der prinzipielle Verlauf der Messung in Abb. 10 mit dem in Abb. 9 vergleichbar ist, zeigen sich Unterschiede insbesondere bei schnellen Änderungen.

Ein prägnantes Beispiel wird am Wochenende der 21. Kalenderwoche 2015 in Dorf im Warndt (Abb. 9) sichtbar: Zunächst wurde die Aufzeichnung am Nachmittag des 22. (Freitag) unterbrochen und am 23. (Samstag) fortgesetzt. Dies war durch Ausstecken der Versorgungsspannung zugunsten eines Kühlwagens bedingt. Nun fand in der Nähe des Messsystems bis zum Sonntagnachmittag ein Fest mit Grill- und Lagerfeuer statt, es zeigen sich ausgeprägte schnelle Signaländerungen. Am Sonntagabend verschwinden diese Einflüsse wieder aus dem Signalverlauf.

\section{Fazit und Ausblick}

Im vorliegenden Modellprojekt konnte gezeigt werden, dass vergleichsweise preiswerte Sensorsysteme auf Basis von Halbleitergassensoren prinzipiell ausreichend empfindlich zur immissionsseitigen Detektion von Belästigungen durch geruchsaktive Substanzen sind. Die zunächst unspezifische Reaktion von Halbleitergassensoren wurde durch den temperaturzyklischen Betrieb und ein trainiertes Auswerteverfahren an die Anwendung angepasst. Sensitivität und Selektivität der Systeme müssen zukünftig weiter verbessert werden. Dazu sollen einerseits analytische Komponenten wie selektive Präkonzentratoren integriert werden, andererseits muss neben dem Abgleich mit Geruchsmeldungen auch durch Referenzmessungen sichergestellt werden,
DOI 10.5162/sensoren2016/5.2.4 dass die stoffliche Erkennung passend zum geruchsrelevanten Gasgemisch erfolgt.

Im Rahmen einer technischen Neukonzeption fließen diese Anforderungen in die Realisierung von Sensorknoten für ein räumlich verteiltes Messnetz ein. Die Sensorknoten werden zur kontinuierlichen, ortsaufgelösten Beobachtung mit einer Mobilfunkdatenanbindung ausgestattet. Damit sollen weitere Feldversuche und Untersuchungen zu Fragestellungen der Immissionsverteilung durchgeführt werden.

Eine wesentliche Entwicklung aus dem beschriebenen Projekt betrifft die Auswertekette, die die Fusion von Winddaten, Bürgermeldungen und Messwerten ermöglicht. Die gefundenen Ansätze werden nun auf Skalierbarkeit untersucht und in eine geeignete Struktur überführt, die sowohl die Anbindung der Eingangsdaten als auch die Weiterverarbeitung der gewonnenen Informationen in GIS-Systemen ermöglicht.

Das Projekt wurde finanziert durch das Ministerium für Umwelt und Verbraucherschutz des Saarlandes. Dank gilt auch den beteiligten Bürgern für ihr großes Engagement.

\section{Literatur}

[1] Peter Boeker: Die Objektivierung des Geruchseindrucks, Messung und Modellierung, Habilitationsschrift, Rheinische FriedrichWilhelms-Universität Bonn, 2003.

[2] VDI-Richtlinie "Olfaktometrie“, VDI 3881, Nr. 1-3 (1986)

[3] T.C. Pearce, S.S. Schiffman, H.T. Nagle, J.W. Gardner (eds.): Handbook of Machine Olfaction - Electronic Nose Technology, WILEY-VCH, 2003.

[4] S. Sironi, L. Capelli, P. Céntola, R. Del Rosso, M. II Grande: Continuous monitoring of odours from a composting plant using electronic noses, Waste Management 27 (3), (2007), 389-397, doi: 10.1016/j.wasman.2006.01.029.

[5] A.P. Lee, B.J. Reedy: Temperature modulation in semiconductor gas sensing, Sens. \& Actuators B 60, (1999), 35-42, doi: 10.1016/S0925-4005(99)00241-5

[6] T. Baur, A. Schütze, T. Sauerwald: Optimierung des temperaturzyklischen Betriebs von Halbleitergassensoren, tm - Technisches Messen, 82 (4), (2015), 187-195; doi: 10.1515/teme-2014-0007

[7] P. Reimann, A. Schütze: Sensor Arrays, Virtual Multisensors, Data Fusion, and Gas Sensor Data Evaluation, in: C.-D. Kohl, T. Wagner (eds.): Gas Sensing Fundamentals, Springer Series on Chemical Sensors and Biosensors, Volume 15, 2014.

[8] VDI-Richtlinie „Wirkung und Bewertung von Gerüchen - Erfassung der Geruchsbelästigung - Fragebogentechnik“, VDI 3883, Blatt 1 (1997) 